\title{
SIMULATION OF AIRFLOW IN GRAIN STORAGE BINS
}

\author{
VANESSA FAORO $^{1}$, Oleg KHATCHATOURIAN ${ }^{1}$, NELSON A. TONIAZZO ${ }^{1}$.
}

\author{
1. Department of Exact Sciences and Engineering, Regional University of the Northwest, Rio Grande do \\ Sul, R. São Francisco, 501, 98700-000, Ijuí Rs, Brazil
}

vanefaoro@yahoo.com.br, olegkha@unijui.edu.br, toniazzo@unijui.edu.br

\begin{abstract}
A mathematical model and software were developed for the three-dimensional simulation of airflow through high capacity grain storage bins by considering the non-uniformity and anisotropy of the seed mass. To validate the proposed model, empirical relationships between air velocity and static pressure drop were obtained for compacted layers of several depths for various grains. A local criterion to estimate the efficiency of complex aeration system in storage bins was proposed. The simulations showed that software could be applied to optimise the performance of existing grain stores and lower the engineering costs of new grain stores.
\end{abstract}

Keywords- Application in Agriculture; 3-D airflow simulation; Aerated grain storage bins; Finite-element method

\section{Introduction}

Aeration is widely used in grain stores to cool the grain mass, to avoid humidity migration, to temporarily conserve the humidity of grains, to remove scents from the grain mass, and to apply fumigation. The resistance to the airflow in an aeration system depends on the airflow parameters, on the characteristics of the product surface (i.e. rugosity), on the form and size of any extraneous impurity in the mass, on the configuration and size of the interstitial space in the mass, on the size and amount of broken grains, and on the depth of the grain. The research carried out by Shedd (1953), Brooker (1969), Haque et al. (1981), ASAE (2000), Navarro and Noyes (2001), Khatchatourian and Savicki (2004), and Khatchatourian and Oliveira (2006), has examined the influence of some of these parameters on airflow pattern in seeds storage. Gayathri and Jayas (2007) have presented a review of the reported mathematical models of airflow through grain mass. In large storage bins, non-homogeneity of grain mass significantly affects flow field. Khatchatourian and Binelo (2008) have researched the compaction of the grain and the airflow pattern under non-homogeneous conditions. As Jayas et al. (1987) and Khatchatourian et al. (2009) showed, there is a strong anisotropy of medium resistance to airflow through grain masses. To simulate the aeration of grain, with any type of air distribution systems, it is necessary to develop software to predict the distribution of the parameters, because obtaining empirical data is a very difficult and costly. Most research on airflow simulation in grain stores is related to one-dimensional, twodimensional or axisymmetric cases; although the flow is usually three-dimensional. Also, the aeration of large grain stores is frequently carried out separately in different segments.

The principal objectives of the present work were: (a) to create a mathematical model, algorithm, and software, to calculate the static pressure, streamlines, and airflow velocity distribution in threedimensions under non-homogeneous conditions; (b) to develop and incorporate into the software a criterion for system performance based on estimating threedimensional air distribution in grain storage bins ; and (c) to carry out numerical simulations of real and hypothetical grain stores with aeration to detect areas of operational risk.

\section{Mathematical model}

Mathematical model presented in detail in Khatchatourian et al. (2009) is reduced to the nonlinear partial differential equation:

$$
\frac{\partial}{\partial x}\left(-k_{x} \frac{\partial P}{\partial x}\right)+\frac{\partial}{\partial y}\left(-k_{y} \frac{\partial P}{\partial y}\right)+\frac{\partial}{\partial z}\left(-k_{z} \frac{\partial P}{\partial z}\right)=0
$$

This equation along with the Dirichlet and Neumann boundary conditions describes the steady-state pressure in a cross-section of aerated grain storage.

\section{Software description and development}

The equation for pressure Eq. (1) was solved by the finite element method using an iterative process to calculate the permeability coefficients $k_{x}, k_{y}$ and $k_{z}$ in each point of the integration domain and using the pressure distribution from the previous iteration step. 
The software, developed in ANSI C++, consisted of tools for geometry construction, mesh generation, generation of system matrix, solver of obtained system of linear algebraic equations and tool for results three-dimensional presentation and analysis.

The geometry of the system can be constructed in any system CAD, CAE, or any 3-D modelling software package that can export data to a standard format. In this work Blender3D was used, (http://www.blender.org). This software is available at no-cost under GPL (General Public License). The storage bin geometry data were exported to smash file format, which is a format that can be read by Tetgen (http://tetgen.berlios.de/). A Perl script (http://www.perl.org/), used for exporting data, was modified to include face materials exportation.

For mesh generation Tetgen, available under GPL license was used. It generates quality tetrahedral meshes using Delaunay algorithms with dynamic adaptive refinement of the mesh.

The developed code is cross-platform and can be compiled in any ANSI C++ compatible compiler. The input files to the solver software are the output files from Tetgen which describes nodes, faces and tetrahedral elements, and generates a file describing the boundary conditions and precision requirements. Using the local matrix information, the global system matrix was generated. The successive over-relaxation (SOR) method was used for resolving the system of linear algebraic equations.

The software executes three iterative processes: 1) it calculates the permeability coefficient in each point of the integration domain, using the pressure distribution in the immediately previous iteration step, 2) it searches the system design point, located in the performance curve of the aerator fan, and 3) it adaptively refines the mesh according to the tetrahedron size per pressure gradient ratio.

\section{Validation of the mathematical model for non-homogeneous conditions in a grain mass}

To validate the proposed mathematical model, the empirical relationships between air velocity and static pressure drop were obtained for compacted layers with several grain storage depths. The empirical coefficients presented in the mathematical model were obtained experimentally for soya bean, maize, rice and wheat grains. In large storage bins, due to compaction, the grain mass was a non-homogeneous medium and the permeability coefficient varied as a function of the grain layer depth as well as pressure gradient. To simulate the aerated grain storage characteristics, the equipment, described by Khatchatourian and Savicki (2004), was used to experimentally determine the grain mass compaction factor caused by the weight of layers above. The influence of compaction on the relationship between the airflow velocity and the static pressure drop were analysed. Experimental data shows the significant influ- ence of the storage layer depth and the airflow direction on the aerodynamic resistance of the grain mass.

\section{Numerical simulations}

Fig. 1 shows the structural layout of real V-form floor storage bin, used in the state of Rio Grande do Sul, Brazil. The storage bin has a maximum width of $30 \mathrm{~m}$ and length of $95 \mathrm{~m}$. Three air inlet systems were analysed: 1) a central inlet system; 2) a system with central and upper lateral inlets; and 3) a system with central, lower lateral and upper lateral inlets. The aeration simulations in storage bins, for different layouts, were generated using the global airflow rate of $Q=9 \mathrm{~m}^{3} \mathrm{~h}^{-1} \mathrm{t}^{-1}\left(2.5 \times 10^{-6} \mathrm{~m}^{3} \mathrm{~s}^{-1} \mathrm{~kg}^{-1}\right)$, which is the most commonly recommended value for aerated grain storage.

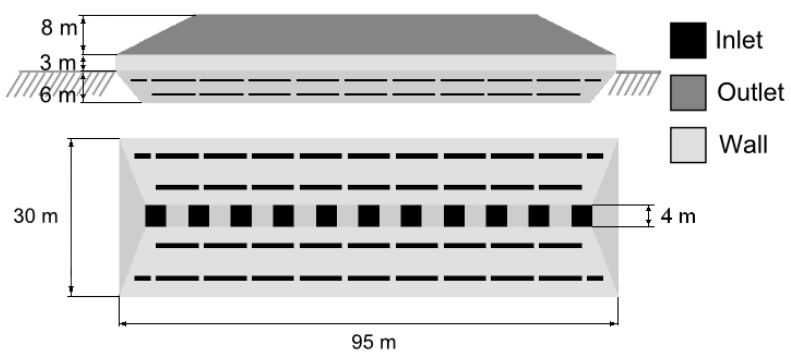

Figure 1. Outline sketch of simulated store bin

Fig. 2(a) shows surface wireframe of the tetrahedral mesh used. The grid had a higher density in regions where the pressure gradient was greater. For the layout under consideration the number of tetrahedrons was approximately 500000.

Analysis of the pressure and velocity distributions showed that the installation of lateral ducts essentially equalised the airflow when compared with the same storage bin without lateral ducts and reduced the initial pressure head. At the same time the directly use of these distributions does not permit to estimate the performance of aeration systems.

5.1 Criterion for describing the efficiency of aeration system.

To estimate the efficiency of an aeration system the specific airflow rate is usually specified. This is obtained from the ratio between the total air flow rate and the total product mass. This criterion is suitable for simple silo designs with constant cross-sectional area, when the air velocity is uniform throughout the storage. If variations in the cross-sectional area are significant, or the aeration distribution system is complex this criterion is not suitable.

To evaluate aeration efficiency for storage bins with variable cross-sectional area and with complex air distribution system, a local specific airflow rate is proposed. For simple storage layouts with constant 
cross-sectional area the specific volume airflow rate $Q$ is:

$$
Q=\frac{Q_{a}}{m_{g}}=\frac{V A}{\rho_{b} H A}=\frac{V}{\rho_{b} H},
$$

where $Q_{a}$ is total air flow rate in $\mathrm{m}^{3} \mathrm{~s}^{-1} ; m_{g}$ is total grain mass in $\mathrm{kg} ; V$ is air velocity in $\mathrm{m} \mathrm{s}^{-1} ; \rho_{b}$ is product bulk density in $\mathrm{kg} \mathrm{m}^{-3} \mathrm{~s}^{-1} ; A$ is the cross-sectional area in $\mathrm{m}^{2} ; H$ is the grain mass depth in $\mathrm{m}$.

The local specific airflow rates for storage bins with the variable cross-sectional area for all internal points $X=X(x, y, z)$ can be presented as:

$$
q_{L}(X)=\frac{V(X)}{\rho(X) L_{X}}
$$

where $q_{L}(X)$ is local specific airflow rate in point $X(x, y, z)$ in $\mathrm{m}^{3} \mathrm{~s}^{-1} \mathrm{~kg}^{-1} ; V(X)$ is air velocity in point $X$ in $\mathrm{ms}^{-1} ; \rho(X)$ is product density in point $X$ in $\mathrm{kg} \mathrm{m}^{-3} \mathrm{~s}^{-1} ; L_{X}$ is full length in $\mathrm{m}$ of a trajectory on which the point $X$ is located.

It is evident that the difference between local value of airflow rate at point and its total average value gives a better estimation of aeration system efficiency than the comparing local and average velocities. For constant cross-sectional area storage bins the value of the local specific airflow rate at all storage points is constant and equal to the value of global specific airflow rate. A local criterion multiplied by aeration time has additive properties. This allows the quality of aeration to be calculated for all parts of the storage bin even if the ventilation is carried out separately at each of the inlets over different periods of time.

To visualise risk domains in the grain storage bin, the distributions of local specific airflow rates were studied for the same grain storage bin with the same global specific airflow rate $Q=9 \mathrm{~m}^{3} \mathrm{~h}^{-1} \mathrm{t}^{-1}\left(2.5 \times 10^{-6}\right.$ $\mathrm{m}^{3} \mathrm{~s}^{-1} \mathrm{~kg}^{-1}$ ).

The number of inputs (from one up to three), their position (upper lateral, lower lateral and central inlets), and ratio of pressure between various inputs were varied. Using the additive property of the local specific airflow rate, estimations of ventilation system efficiency were carried out separately using each of inlets during the different periods of time. Relationships between the duration of ventilation time through each inlet and the airflow rates were chosen so that the global specific airflow rate $Q$ was equal to $9 \mathrm{~m}^{3} \mathrm{~h}^{-1} \mathrm{t}^{-1}$.

As the simulation results show, if only one airflow inlet is used, there is always a large area with superfluous ventilation. Since the airflow tends to leave the grain mass through the line of least resistance, moving the airflow inlet from the lower position to the upper position provokes a pressure reduction and a deterioration in ventilation uniformity. Therefore, there is area with excessive ventilation close to the upper lateral inlet if all of three inlets operate togeth- er with identical pressure. By selecting the appropriate pressures ratio for the inlets it is possible to considerably improve the system of air distribution in the storage bin. The system with central, lower lateral and upper lateral inlets considerably improved the conditions of storage in regions close to walls when compared with other inlet systems.

In high capacity storage, the grain ventilation is usually carried out stage by stage, serially using air inlets located in different storage sections. Under these conditions the advantage of using of local specific airflow rates for ventilation efficiency estimation is especially great.

The simulations indicate the significant advantage of ventilation carried out in turn by each of inlets in compared with the simultaneous use of all inlets at equal pressures. This improvement is caused because the capacity for air to penetrate to all zones under the dominant influence of each inlet results in a more uniform distribution of $q_{L}$. By varying the duration of aeration for each inlet, it is possible to find a optimum distribution of $q_{L}$ for a given storage bin design.

For example, Fig. 2( $a, b)$ shows the distribution of $q_{L}$ in the grain storage bin with alternate use of upper lateral, lower lateral or central inlets for durations of aeration varying as $t_{1}: t_{2}: t_{3}=1: 1: 1$ (a) and $t_{1}: t_{2}: t_{3}=1: 2: 2(b)$. As results showed, the last distribution had the fewest regions with insufficient or excessive aeration. These regions are shown in Fig. $3(b, c)$ for whole grain storage bin.

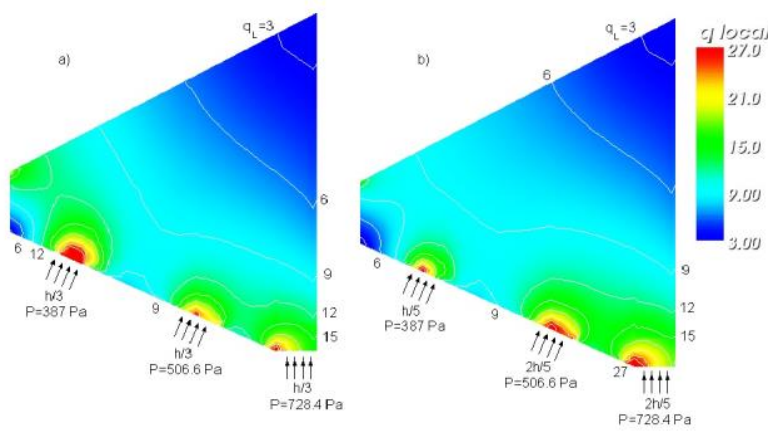

Figure 2. Distribution of resultant local specific airflow rate with separated functioning inlets, $\mathrm{Q}=9 \mathrm{~m}^{3} \mathrm{~h}^{-1} \mathrm{t}^{-1}$ : a) upper lateral, lower lateral and central inlets with equal application times $(1: 1: 1)$; b) upper lateral, lower lateral and central inlets with different application times $(1: 2: 2)$ 

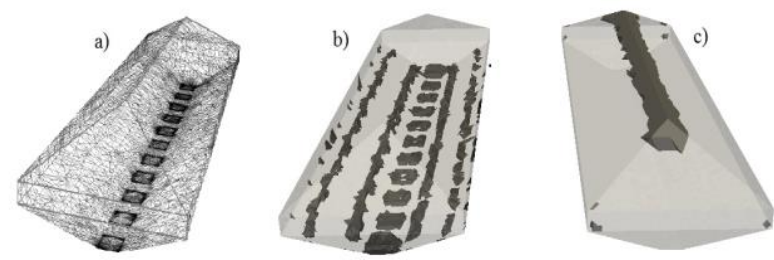

Figure 3. Surface wireframe of the tetrahedral mesh (a) and visualization of domains with the lowered intensity of ventilation $q_{L}<4.5$, (c); and with excessive intensity of ventilation, $q_{L}>18$, (b); $q_{L}$ was obtained by superposition of simulations for separated functioning of upper lateral, lower lateral and central inlet with different application times $(1: 2: 2) ; \mathrm{Q}=9 \mathrm{~m}^{3} \mathrm{~h}^{-1} \mathrm{t}^{-1}$

\section{Conclusion}

A mathematical model of 3-D airflow in an aerated grain storage system was developed for nonuniform conditions of the seed mass. Experiments were conducted to obtain the relationship between air velocity and pressure gradient and the values of the porosity factors for different seed type and different storage layer depths. A local criterion was proposed to estimate the efficiency of complex aeration system in grain storage bins. Software was developed to determine the velocity, pressure and local specific airflow rates distributions, the global airflow rate or initial pressure head in the grain mass store for 3-D cases.

The aeration system efficiency of several stored seeds was analysed to provide the airflow distribution uniformity and the static pressure head values that generate the appropriate airflow rate for safe storage. It was shown that the aeration system of grain storage bin can be essentially improved by the use of inlets system with different initial pressures selected for each inlet. Also, it was shown that is possible to optimise air distribution in a grain storage bin by operating each inlet in turn and by selecting a suitable aeration period for each inlet.

\section{Acknowledgements}

The authors would like to thank $\mathrm{CNPq}$ for the financial support for this work (process $\mathrm{N}^{\circ}$ 313706/2009-3).

\section{References}

ASAE (2000). Resistance to airflow of grains, seeds, other agricultural products, and perforated metal sheets, American Society of Agricultural Engineers, St. Joseph, MI

BROOKER D B (1969). Computing air pressure and velocity distribution when air flows through a porous medium and nonlinear velocity - pressure relationship exists. Transaction of the ASAE, 12, 118-120 DOI: $10.13031 / 2013.38776$

GAYATHRI P; JAYAS D S (2007). Mathematical modeling of airflow distribution in grain bulks a review. ASAE Annual Meeting 076226, 1-12

HAQUE E; CHUNG D; FORSTER G (1981). Pressure and velocity field in airflow through packed bed of corn with fines non-Darcy flow conditions. Transactions of the ASAE, 5, 1595-1604 DOI: $10.13031 / 2013.34496$

KHATCHATOURIAN O A; TONIAZZO N A; GORTYSHOV Y F (2009). Simulation of airflow in grain bulks under anisotropic conditions. Biosystems Engineering, 104(2), 205-215

DOI: 10.1016/j.biosystemseng.2009.06.023

KHATCHATOURIAN O A; BINELO M O (2008). Simulation of three-dimensional airflow in grain storage bins. Biosystems Engineering, 101(2), 225-238 DOI: 10.1016/j.biosystemseng.2008.06.001

KHATCHATOURIAN O A; DE OLIVEIRA F A (2006). Mathematical modelling of airflow and thermal state in large aerated grain storage. Biosystems Engineering, 95(2), 159-169 DOI: 10.1016/j.biosystemseng.2006.05.09

KHATCHATOURIAN O A; SAVICKI D L (2004). Mathematical modelling of airflow in an aerated soya bean store under non-uniform conditions. Biosystems Engineering, 88(2), 201-211 DOI: 10.1016/j.biosystemseng.2004.03.001

JAYAS D S; SOKHANSANJ, S; MOYSEY E B; BARBER E B (1987). The effect of airflow direction on the resistance of canola (rapeseed) to airflow. Canadian Agricultural Engineering, 29, 189-192

NAVARRO S; NOYES R T (eds) (2001). The Mechanics and Physics of Modern Grain Aeration DOI: $10.1201 / 9781420040333$ Management. CRC Press LLC. USA

SHEDD C K (1953). Resistance of grains and seeds to air flow. Agricultural Engineering, 34(9), 616-619 